\title{
ÉTABLISSEMENT D'UN \\ MODÈLE MATHÉMATIQUE HYDROLOGIQUE SERVANT A \\ LA PRÉVISION ET L'ANNONCE DES CRUES DU \\ RIO GUADALQUIVIR (ESPAGNE)
}

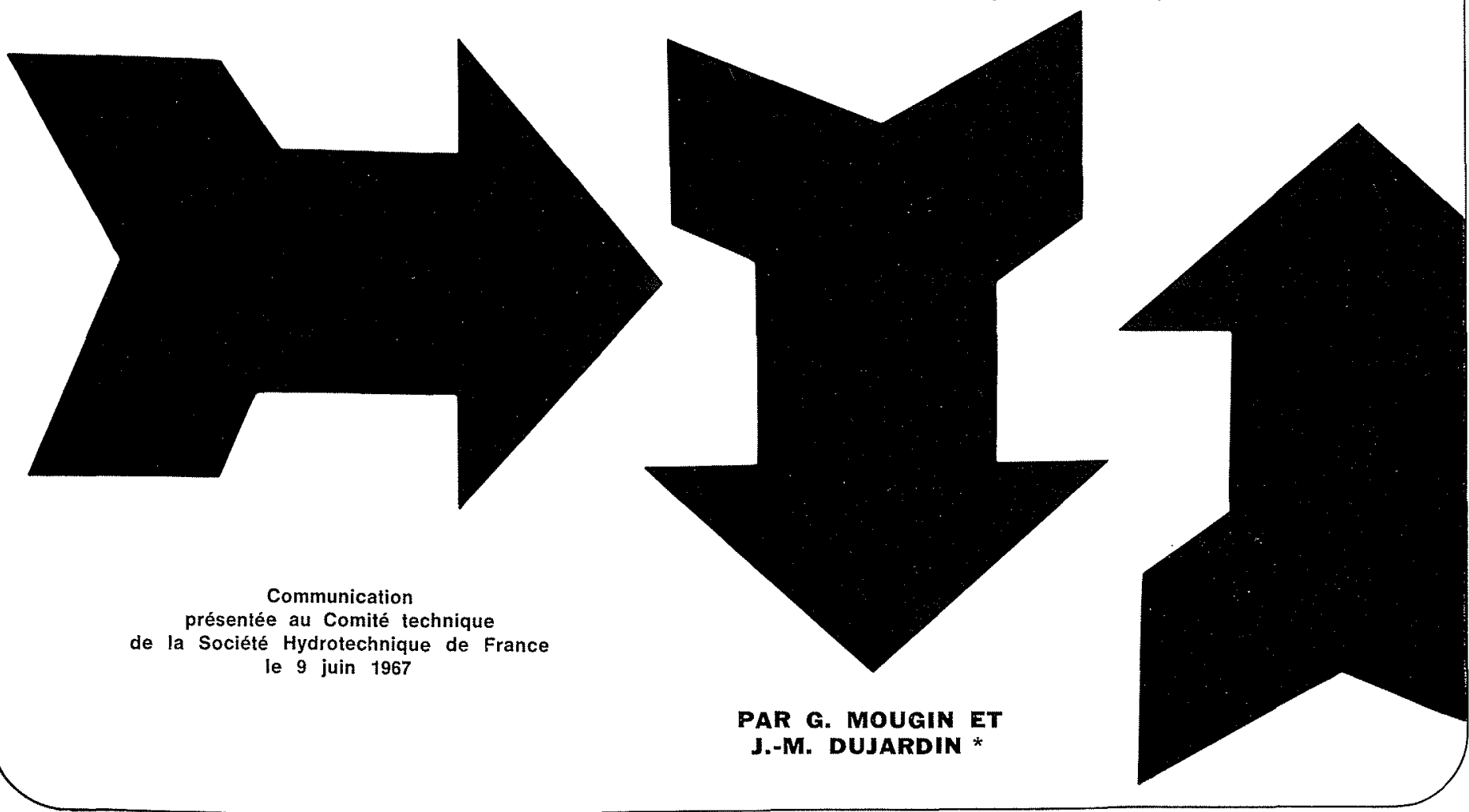

Idées directrices qui ont présidé à la conception du modèle mathématique hydrologique

1. Les études ont pour mobile la recherche de la protection de la région de Séville contre les crues, par les différents moyens :

- de l'annonce des crues;

- de la défense contre les inondations;

- du contrôle des crues.

L'annonce des crues demande que soient connues les conditions particulières de la propagation des intumescences dans le cours d'eau considéré.

La défense contre les inondations, procurée par l'aménagement du lit et de la zone inondable, a une incidence sur les conditions d'écoulement des crues, entrainant un remaniement de l'annonce des crues.

Le contrôle des crues peut devoir son efficacité à la manœuvre appropriée de barrages, en se basant sur la prévision des apports à ces barrages.

2. La simulation de la genèse et de la propagation des crues en amont de Séville, à l'aide d'un modèle mathématique, est le procédé d'étude qui s'applique par excellence au problème à faces multiples ainsi posé des crues du Guadalquivir.

L'étude des moyens de défense contre les inon-

\footnotetext{
* Ingénieurs à la SOGREAH, Grenoble.
}

dations et de contrôle des crues comporte nécessairement un aspect économique, car, d'une part, la rentabilité de l'association de ces deux moyens est à examiner et, d'autre part, les barrages n'ont pas la vocation exclusive de lutte contre les crues.

En conséquence, le modèle mathématique hydrologique est le moyen nécessaire pour reconnaitre l'efficacité possible des aménagements hydrauliques dans la lutte contre les crues, mais il doit être complété par une recherche de l'optimum économique d'exploitation des barrages. Le modèle mathématique hydrologique se présente donc comme une pièce importante de l'outil que réclamerait la planification du développement hydraulique du bassin du Guadalquivir.

La construction du modèle mathématique hydrologique a été conçue de sorte à utiliser dans toute la mesure du possible les données déjà disponibles sur les pluies, les crues, les barrages et la topographie. Ceci afin de donner l'occasion de mettre en ordre, de critiquer et de valoriser le stock volumineux des données existantes.

Egalement, l'entreprise du modèle pour l'étude des crues permet de déceler les lacunes dans les données existantes et d'orienter en conséquence le programme de réhabilitation du réseau des stations hydroclimatologiques, voulu pour le bassin du Guadalquivir.

Les données disponibles pour entreprendre l'étude des crues dans les directions qui viennent d'être rappelées, ont conduit à considérer finalement la partie du bassin que parcourt le Guadal- 
quivir, entre les barrages de Doña-Aldonza en amont et d'Alcala-del-Rio en aval. Le tronçon du fleuve, délimité par ces deux aménagements hydroélectriques au fil de l'eau, a $400 \mathrm{~km}$ de longueur.

\section{Conception du modèle.}

\section{Annonce des crues. Modele fondamental.}

L'annonce des crues à Séville, à partir des cotes d'eau observées en différents points du bassin versant en amont, est le moyen le plus précis de prévoir l'ampleur des inondations menaçant la ville. Il s'agit essentiellement de calculer la composition des crues repérées sur les affluents avec l'onde de crue se propageant le long du Guadalquivir.

Ces calculs de la composition et de la propagation vers l'aval d'ondes de crues connues en un certain nombre de points du fleuve constituent en quelque sorte le squelette du modèle mathématique à l'étude. Dans la suite, cette partie fondamentale du modèle mathématique d'ensemble sera appelée modèle fondamental.

Le modèle mathématique fondamental n'est que le premier rouage de l'instrument final. Sa fonction est de reproduire la propagation des crues, en supposant connus à chaque instant les débits des afluents, les manœuvres des barrages au fil de l'eau, et les conditions aux limites amont et aval. Moyennant certaines transformations, ce modele servira à l'annonce des crues, mais non à Ieur prévision.

\section{Prévision des crues. Modèle définitif.}

Il faudra donc améliorer le délai de l'alerte aux crues en effectuant une véritable prévision de crue, à moyen terme, c'est-à-dire augurer de la gravité des inondations à Séville dès que des précipitations importantes se manifestent sur le bassin versant.

A cet effet, la partie importante et originale du modèle mathématique sera constituée par un système de calculs, qui réalisera la transformation des débits pluviaux en hydrogrammes des crues, là où ces hydrogrammes doivent être fournis au modèle fondamental pour le calcul de propagation des ondes jusqu’à Séville.

\section{Etapes déjà franchies de l'étude Résultats acquis}

Les études, commencées en janvier 1964, comportent plusieurs aspects assez différents el complémentaires.

\section{Le rassemblement et l'élaboration des données existantes sélectionnées pour l'étude des crues:}

a) Pluies journalières mesurées dans la période octobre-avril de 1953 à 1963 , en plus de 400 postes pluviométriques;

b) Niveaux et débits partout mesurés sur Ies affluents depuis 1953 , et données sur la manouvre des barrages du Guadalquivir, effectuée lors du passage d'une dizaine de grandes crues depuis 1951; c) Reconnaissances détaillées du terrain, préparation des travaux topographiques complémentaires (lever du profil en long du fleuve et de près de 200 profils en travers du lit el du champ d'inondation, entre Doña-Aldonza el Alcala-del-Rio), évaluation sur place des rugosités;

d) Recensement des onvages de toute nature qui provoquent une perte de charge singuliere dans le lit du fleuve;

e) Rassemblement des documents photographiques et topographiques existants;

f) Exécution des travaux topographiques complé. mentaires.

\section{Construction et réglage du modèle fondamental.}

a) La propagation des intumescences dans les canaux el rivières est représentée par les équations de Saint-Venant dont la résolution est entreprise par une méthode aux différences finies, en découpant la rivière en un certain nombre de troncons de longueur $\Delta x$ et en calculant l'état de la rivière en des instants séparés par un intervalle du temps $\Delta t$.

SOGREAH a choisi pour le Guadalquivir la méthode «implicite» qui consiste à établir en tout point de calcul une loi hauleur-débit différentielle en partant du point aval où la condition limite est généralement connue sous cette forme jusqu'à l'amont où une autre condition aux limites permet, par un calcul à rebours vers l'amont, d'expliciter les variations de niveau $d y$ et de débit $d q$.

La transmission normale de la loi peut ètre perturbée par des accidents (barrages, affluents) qui sont à traiter à part (points spéciaux). Le troncon du Guadalquivir représenté, du barrage de DoñaAldonza à l'amont jusqu'à celui d'Alcala-del-Rio à l'aval, soit $400 \mathrm{~km}$, est représenté par 88 points normaux et 55 points spéciaux (amont et aval de la singularité), ce qui fait 198 points de calcul.

b) La construction du modèle exige des matériaux topographiques (sections transversales du lit, zones inondées, etc.) et des malériaux hydrauliques (rugosité, pertes de charge singulières, barrages mobiles). Iue réglage du modèle consiste, une fois les matériaux topographiques mis en place, à corriger la marge subjective des matẻriaux hydrauliques jusqu’à ce que les phénomènes reproduits coîncident avec les phénomènes réels.

Le réglage par reproduction d'étals permanents étail impossible sur le Guadalquivir. La multiplicité des lâchers de barrages et des pompages ainsi que l'irrégularité naturelle des apports ne permetlent pas d'imaginer un état permanent. Mais il était possible de régler les paramètres hydrauliques on essayant de reproduire le passage d'états non permanents comme les crues, pendant lesquelles l'état du fleuve en fonction du temps a été observé en un certain nombre de points. Ceci est possible à condition que l'on connaisse à chaque instant toutes ies contraintes variables, à savoir : débits des affluents, manœurres des barrages, conditions limites amont et aval.

Le critère important du réglage «par crue» est en définitive le respect du temps de propagation des ondes de crue. Le réglage est réputé satisfaisant 
lorsque le modèle reproduit correctement les temps de propagation et les niveaux réellement observés pour plusieurs crues de forme et d'amplitude variables. Parmi les crues dont les données ont été rassemblées et étudiées, quatre ont été retenues pour régler le modèle. Un premier calcul est effectué avec les données reconnues valables et l'on s'emploie ensuite à corriger les anomalies que le calcul présente par rapport à la réalité. Il a falíu en particulier reconstituer à partir des précipitations les apports de certains afluments non dotés de stations de mesure.

c) Le réglage du modèle fondamental, effectué au micux des données hydrologiques existantes, est satisfaisant pour la reproduction des grandes crues. Il a entrainé une première critique des données existantes et a permis de rectifier la valeur jusqu'alors admise pour la crue catastrophique de février 1963. Ce modèle ainsi réglé permet de simuler le déroulement des grandes crues jusqu'à Alcaladel-Rio quand les débits sont connus à DoñaAldonza et sur les affluents en aval.

\section{Exploitation systématique des données sur les averses.}

Il s'agissail d'opérer, parmi les postes pluviométriques en service depuis de nombreuses années, une sélection telle que les postes pluviométriques sélectionnés :

- suffisent pour évaluer l'apport pluvial d'une averse qui engendre une crue notable d'un tributaire du Guadalquivir;

- soient en nombre minimal pour faciliter le fonctionnement d'un résean de prévision des crues et rendre son écuipement aussi économique que possible.

L'étude de 405 postes pluviométriques, répartis en 59 bassins élémentaires sur une période de 70 mois pluvieux, ce qui représente 850000 informations pluviométriques journalières, a été faite sur ordinateur électronique, par un calcul de corrélation entre les volumes d'averses mesurés aux différents postes pluviométriques d'un même hassin. Dans le cas où nous possédions des données sur les crues à l'exutoire, des corrélations ont également été faites entre le volume de l'averse en un poste pluviométrique et le volume de la crue à l'exutoire du bassin considéré. L'opération a abouti à la sélection de 58 postes représentatifs retenus pour la détermination d'indices pluviométriques d'alerte aux crues. Ces indices donnent le moyen de déclencher, en temps opportun, le dispositif d'annonce des crues qui peuvent être alarmantes pour la région de Séville.

\section{Essais systématiques effectués sur le modèle fondamenta en vue d'étudier l'atténuation possible des crues par les barrages d'accumulation situés sur les affluents,}

L'étude est limitée aux six barrages actuellement en service et tous situés sur les affluents rive droite; les manœuvres imaginées sont affranchies de toute prévision, c'est-à-dire que l'on suppose connus à l'avance les hydrogrammes des crues sur les affluents et que l'on cherche quelles seraient les possibilités d'écrêtement maximal de la crue résultante du Guadalquivir en fonction du creux réservé dans les retenues et de différentes consignes de manœuvres aux barrages.

Les limites de ces possibilités d'écrêtement étaient fixées par la «crue sauvage » qui se serait produite si aucun barrage n'avait existé et par la «crue minimale $\gg$ qui se serait produite si les six barrages pouvaient retenir toute la crue de l'affluent considéré.

Il est apparu que les consignes actuelles d'exploitation des barrages donnaient déjà de bons résultats. Le creux réglementaire maintenu dans la retentie, conditionné par les nécessités de l'irrigation, s'avère généralement suffisant pour obtenir une atténuation maximale si les consignes sont judicieusement choisies et les consignes actuelles de manouvre, absorption complète dès le début de la crue jusqu'au remplissage de la retenue, s'avèrent ctre assez eflicaces pour une utilisation rationnelle du creux initial.

Il n'en reste pas moins que l'exploitation prévue de nouvelles retenues ne pourra que contribuer à l'atténuation des crues avec des consignes qu'il faudra étudier de facon plus précise et que l'atténuation oplimale de la crue du Guadalquivir ne sera assurée dans la pratique que lorsque les apports en certains des barrages sur les affluents seront prévisibles à partir des pluies.

\section{Campagne de mesure.}

L’étude de la prévision à partir des pluies des apports aux barrages a donné l'occasion de procéder à une nouvelle critique des donnces hydrologiques. Elle a confirmé la nécessité absolue qu'il y avait de lancer la mise sur pied d'un réseau de stations hydroclimatologiques. Nécessité qui se fait sentir non seulement pour la prévision des crues, mais également pour élargir le domaine d'emploi du modèle fondamental, en tant qu'instrument de simulation des crues de toute importance, intégré dans un modèle économique.

Le programme comportail la réhabilitation ou l'installation d'un réseau comportant 45 postes d'observations détaillées des averses et 32 stations hydrométriques. Le Iancement de la construction et de l'équipement a démarré en novembre 1965. Pour l'étude des crues, le dispositif de mesures n'a besoin de fonctionner que de novembre à avril, mais lorsqu'on envisagera d'utiliser également l'instrument de simulation des écoulements pour d'autres études, il sera sans doute nécessaire de faire porter la campagne de mesures sur les 12 mois de l'année.

Jusçu'à présent, la campagne de mesures en reste à la phase de démarrage et de mise au point des moyens nécessaires. Elle reste donc un des objectifs principaux pour la suite des études.

\section{Prévision à partir des pluies des apports journaliers.}

Les résultats des mesures entreprises au cours de l'hiver 1965-1966 et notamment cenx portant sur les crues de janvier et février 1966 ont permis de reprendre sur des bases plus solides l'étude de la prévision des apports des crues aux barrages à partir des averses.

I.es bassins considérés ont une surface comprise entre 1000 et $2500 \mathrm{~km}^{2}$ et le temps de concentration n'y est pas supérieur à $24 \mathrm{~h}$. La consistance 
des données disponibles ne permet qu'une prévision journaliere des apports selon une formule

où :

$$
\mathrm{V}_{j+1}=\alpha \cdot \mathrm{V}_{j}+\mathrm{C} \cdot \mathrm{P}_{j}
$$

$\mathrm{V}_{j}$ est le volume des apports du jour $\mathbf{J}$;

$P_{j}$ est l'indice pluviométrique du bassin (hauteur de pluie moyenne le jour $J$ );

$\alpha$ est un coefficient d'écoulement différé;

$C$ est un coefficient d'écoulement rapide, défini comme une fonction de l'indice des précipitations antéricures tel qu'il est utilisé par Linsley en particulier el que nous utilisons sous la forme

$$
\mathrm{I}_{\mathrm{I}}=\left(\mathrm{I}_{j-1}+\mathrm{P}_{j-1}\right) \times 0,85 \text {. }
$$

Cet indice étant limilé par un maximum qui correspond à l'état de saturation du sol el qui peut varier d'une saison à l'autre el d'un bassin à un autre.

Il est maintenant acquis que la méthode générale de transformation pluie-ruissellement ainsi définie pourra être utilisée avec profit, lorsque le temps de réponse des bassins n'est pas sensiblement inférieur à $24 \mathrm{~h}$, mais que seule la poursuite de la campagne de mesures permettra d'obtenir le volume de données nécessaire pour mettre au point les valeurs paramétriques de la formule de prévision.

\section{Instrument pratique d'annonce des crues.}

Exploration des possibilités qui s'offrent à la dérivation, à partir du modèle hydrologique actuellement construit, d'un instrument pratique d'annonce des crues, c'est-à-dire d'un modèle simplifié de propagation des crues, destiné à être opéré en «temps réel ». Plusieurs solutions, possibles a priori, ont été examinées :

-. simplification du modèle fondamental, sans en changer le principe;

- adoption d'une autre méthode de calcul, plus expéditive et basée sur un principe radicalement différent et qui serait réglée grâce au modèle fondamental.

La propagation des crues dans les grandes rivières relève souvent davantage d'une analyse des volumes transités el accumulés que d'une analyse des facteurs de vitesse et d'inertie et l'analyse des équations est dans ce cas considérablement plus simple, de sorte que nous nous sommes orientés vers une méthode de flood-routing et parmi celles-ci la méthode «Muslingum » a paru la mieux adaptée au cas du Guadalquivir. Elle permet de ramener le cours du Guadalquivir à une quinzaine de troncons et de calculer l'hydrogramme à l'aval d'un troncon à partir de l'hydrogramme rentrant à l'amont, après le réglage, à l'aide du modèle fondamental, des temps de propagation et des coefficients d'amortissement dans le troncon.

Cette seconde méthode parait mieux adaptée au problème de l'annonce des crues et constituerait un opérateur qui pourrait ètre mis en ouvre par calcul digital ne requérant qu'un ordinateur de faible puissance, ou par emploi manuel d'abaques ou au moyen d'un système analogique électrique ou hydraulique.

Techniquement parlant, une méthode expéditive de calcul, programmée pour une machine de petite puissance offrirait le plus d'avantages, notamment en matière d'introduction des données. Toutefois, il convient de considérer non sculement l'angle technique du traitement des données, mais aussi d'autres facteurs importants qui pèsent dans le choix de la solution la plus avantageuse : cô̂t et sécurité de la transmission des données depuis le terrain, taux d'occupation et frais d'exploitation du matériel; frais de premier investissement et possibilité d'amortissement du matériel par une affectalion à buts multiples, ete. Ces différents points restent à examiner pour choisir la voie dans laquelle sera entreprise la mise au point du système pratique d'annonce des crues.

\section{Orientations possibles de études selon les objectifs finalement visés}

1. De toute façon, le besoin de données hydrologiques nouvelles est impérieux el l'entreprise d'une solide campagne de mesures s'inscrit en tête des études à poursuivre. Seulement, la taille à donner au dispositif de mesures n'est pas la même selon que le modèle hydrologique est destiné finalement à servir un modèle économique ou seulement à obtenir un système de prévision des crues. La structure du dispositif de mesures ménage la possibilité de pousser le modèle hydrologique jusqu'à la simulation des écoulements que demanderait un modèle économique.

Mêmes vues sous l'angle exclusif de la prévision des crues, les études déjà accomplies auraient été nécessaires. Un modèle hydrologique basé sur tout autre principe, par exemple sur celui d'une transformation pluie-ruissellement, globalement effectuée pour le bassin en amont de Séville, n'aurait pas convenu. Le bassin du Guadalquivir comporte déjà un grand nombre de retenues artificielles capables de déformer sensiblement, et de façon très variable, les caractéristiques stables de l'accumulation naturelle des eaux sur le bassin. Les essais systématiques, de portée pourtant limitée, qui ont été effectués sur le modèle fondamental, ont d'ailleurs démontré que les crues arrivant à Séville sont déjà nettement influençables par les quelques retenues qui existent sur les affluents de rive droite. Il fallait donc expliciter dans le modèle hydrologique le rôle possible des différents barrages, et entreprendre nécessairement la construction du modèle fondamental, en réservant les transformations pluie-ruissellement aux bassins des affluents.

2. Dans l'hypothèse où l'objectif des études se bornerait finalement au seul établissement d'un système pratique de prévision et d'annonce des crues, les études déjà faites seraient à compléter seulement sur les points suivants :

- campagne de mesures mettant en œuvre une partie seulement du résean des stations hydroclimatologiques en cours d'installation. Ce dernier a été conçu pour servir aux études plus étendues, poursuivies jusqu'à présent. L'élagage à faire maintenant dépendrait des points du 
Guadalquivir où la prévision des crues serait désirée;

- mise au point de la structure du système pratique d'annonce des crues à partir du modèle fondamental tel qu'il est actuellement réglé, et du modèle simplifié du type «Muskingum»;

--- mise au point des valeurs paramétriques qui interviennent dans la méthode de prévision des apports des affluents à partir des pluies. La campagne de mesures servirait également à cet effet;

- étude de l'appareillage qui assurera la transmission et le traitement des informations;

- étude de l'organisation du réseau des stations de prévision et d'annonce des crues.

3. Dans l'hypothèse où le modèle hydrologique devrait être poursuivi dans le but de servir également à l'établissement éventuel d'un modèle éco- nomique, - et les études ont été engagées jusqu’à présent dans ce sens -, les études à poursuivre, axées sur le système pratique de prévision des crues dérivables du modèle fondamental actuel, devraient de plus être élargies sur les points suivants :

- campagne de mesure requérant la mise en service de la totalité du réseau hydroclimatologique conçu au cours des études;

- entreprise du fin réglage du modèle fondamental, actuellement réglé pour les seules crues importantes, quand la campagne de mesures aura produit des données adéquates;

- le modèle fondamental, une fois affiné, constituerait l'instrument de simulation approprié à la recherche des moyens de lutte contre les inondations, à l'étude de la production d'énergie au passage des crues et à l'étude des crues artificielles provoquées par de brusques manœuvres aux barrages.

\title{
Abstract \\ Construction of a hydrological mathematical model for a Rio Guadalquivir flood warning and forecasting study
}

\author{
by G. Mougin * and J.-M. Dujardin **
}

\section{Basic model design concepts.}

The object of the investigations was to find ways of protecting Seville against floods by the following means:

(i) Flood warning by calculation of river level rise propagation;

(ii) Medium-term flood forecasting by a rainfall/runoff transformation calculation for the tributary basins;

(iii) Flood protection by suitable structures of the river bed and floodable area, and the effect of such works on flow conditions;

(iv) Flood control by appropriate dam operation, based on foreseeable inflows at the dams.

Simulation of flood formation and propagation by a hydrological mathematical model is the means required to ascertain the potential effectiveness of hydraulic works against floods. If combined witl an economic probability study of the flood prevention works and an operational research study of optimal dam operation, the model would be an important part of the means required in planning lydraulic development in the Guadalquivir basin.

\section{Progress and results to date.}

The first steps in the study, which started in 1964, were to collect and prepare any available data on rainfall, floods, dams and topography, to evaluate frictional resistance to flow, to list structures causing local head losses, etc.

The basic propagation model was constructed by the implicit solution method for the Saint Venant equations. The model represents a $400 \mathrm{~km}$ stretch of the Guadalquivir from the Dona Aldonza dam upstream to the Alcala del Rio dam downstream. The model was adjusted by running a certain number of observed floods on it; it can be used to simulate major floods all the way down to the Alcala del Rio dam if the upstream and downstream tributary flow rates are known.

The model adjustment showed up gaps in the data and helped to guide the rehabilitation of the hydroclimatological observation network in the basin. As a result, the construction and equipping of 32 gauging stations and 45 detailed shower observation stations was put in hand and a field measurements programme was organised.

After systematic application of shower data already available from 405 stations, 58 were picked out as representative for the determination of critical rainfall indices for flood warning purposes, enabling the Seville flood warning system to be set off in good time (floods in the Seville region are apt to assume quite alarming proportions at times).

Systematic tests carried out with a view to studying flood attenuation by storage dams on tributaries showed that

\footnotetext{
* Ingénieurs à la SOGREAH, Grenoble.
} 


\section{G. MOUGIN et J.-M. DUJARDIN}

present dam operating rules (calling for some storage space to be kept available in the reservoir and the latter to be made to absorb floods fully from the moment they start until the reservoir is full) are already giving good results. In practice, however, optimum attenuation of the Guadalquivir flood will not be ensured until the inflows at certain dams ont the tributaries can be predicted from the rainfall data.

Although the field measurements programme is only just starting, it has already vielded data which have enabled a start to be made with a study of daily dam inflow prediction from rainfall data. A general rainfall/discharge transformation method has been produced, and further field measurements are expected to provide the data required to establish final values for the parameters in the prediction formulae.

Consideration of possible ways of developing a simple practical flood forecasting instrument from the present hydrological model has led to the adoption of the more expeditious Muskingum calculation method, which is based on volume analysis and can be ajusted by the basic model. This method seems to be better matched to the flood forecasting problem and could be applied by digital computation requiring no more than a computer of limited capacity, or by an electrical or hydraulic analogy system.

\section{Possible subsequent lines of investigation.}

Further investigation could be confined to development of a practical flood forecasting and warning system, but the hydrological model has also been designed to serve for the construction of an economic study model if required. In any case, there is urgent need for fresh hydrological data, and priority should be given to a thorough field measurements programme. Requirements for a study confined to forecasting will be to put the finishing touches to a practical flood forecasting system, to establish final values of the parameters in the inflow prediction method, and to study organisation of the forecasting and warning network and the necessary data-transmitting and processing facilities. For a study with a wider scope, the basic model (now only calibrated for major floods) will require fine adjustment in order to provide a suitable means of simulation for use in studies of a general nature.

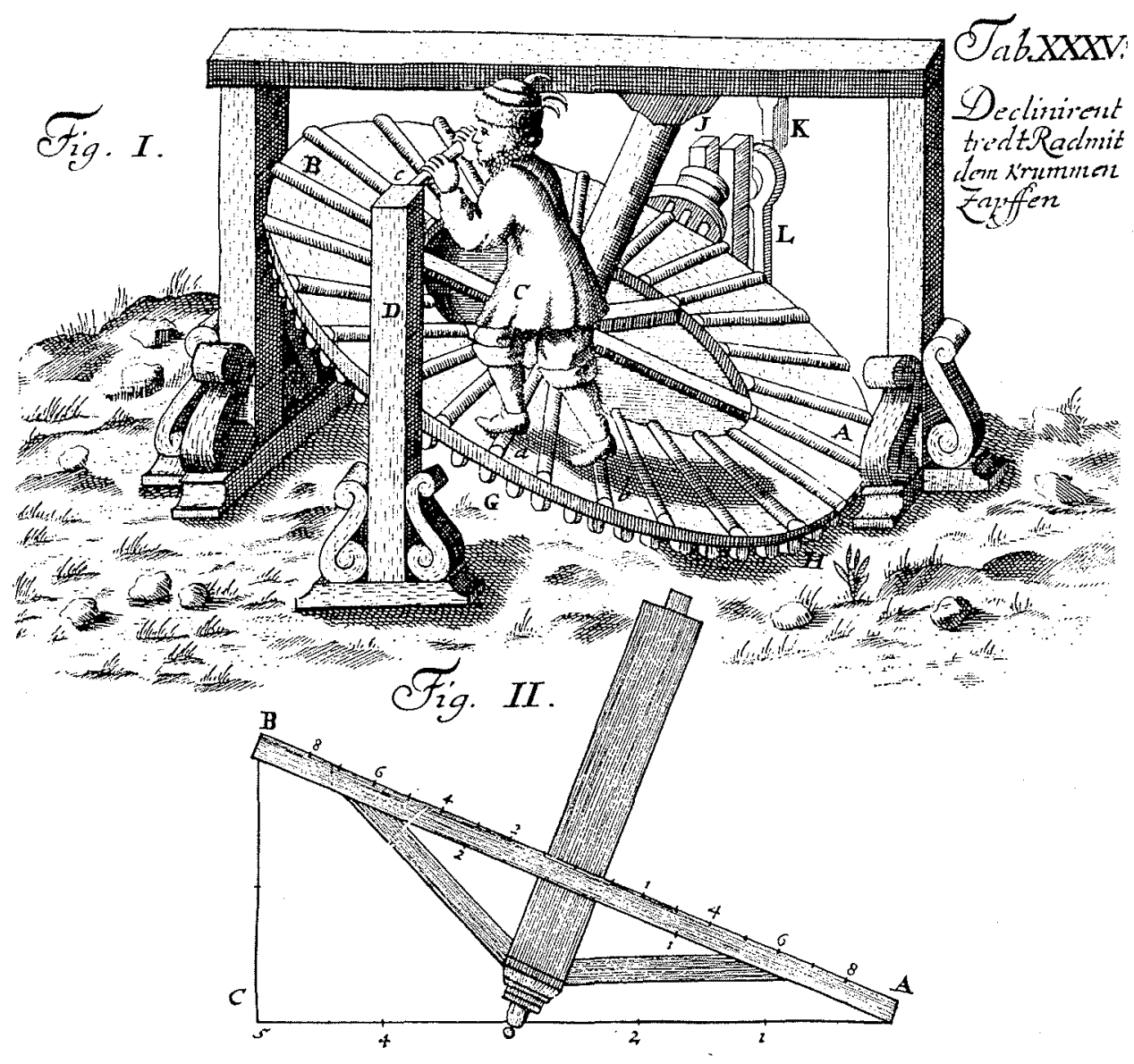

